\title{
Wanted: manufacturer for Ebola and Marburg vaccines
}

Now that the excitement over $\infty$ the discovery has subsided, the hard work of bringing to market the world's only vaccines for Ebola and Marburg hemorrhagic fever is just beginning.

Dr. Steven Jones, a research scientist at the Winnipeg-based National Microbiology Labora-

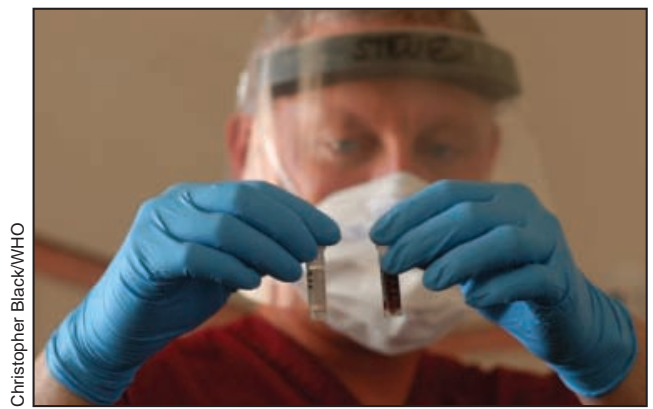

Winnipeg's Dr. Steven Jones tests a blood sample for Marburg fever in Uige, Angola. tories and a codiscoverer of the vaccines, said the search is on for a private-sector contractor to conduct human trials, which may take up to 18 months, and manufacture the vaccines.

In June, Jones and his colleagues - Dr. Heinz Feldmann of Winnipeg and Dr. Thomas Geisbert of the US Army Research Institute for Infectious Diseases at Fort Detrick, Maryland - announced that they had successfully vaccinated monkeys against the deadly Ebola virus and genetically related Marburg virus (Nature Medicine 2005;11:786-90).

Jones says the Marburg vaccine is based on the Musoke strain and cross protects across strains. The Ebola vaccine is based on the 1976 strain of the Zaire species and protects from the 1995 , but not the other 2 species that affect humans.

There is no treatment for Ebola or Marburg fever, which causes diffuse vascular permeability and bleeding. It kills between $40 \%$ to $90 \%$ of those infected. Since 2004, Angola has experienced an outbreak of Marburg, which, as of July 29, had killed 323 of the 368 infected, according to WHO.

Jones and his colleagues took some of the prototype vaccine with them on a recent trip to Angola in case of a needle- stick injury. Of 86 needle-stick involving Ebola or Marburg virus and medical staff, there have been no survivors, Jones said. "That makes [using the vaccine] a very easy decision." They didn't need it. - Dan Lett, Winnipeg

National Initiative

\section{Safety campaign aims to reduce adverse events}

About half of Canada's health care organizations have enrolled in a patient safety campaign aimed at reducing the number of adverse-event related deaths and injuries in hospitals.

Launched on June 28, the Canadian Institute for Patient Safety's Safer Healthcare Now! (www.saferhealthcarenow.ca) campaign has already enrolled 95 health care institutions and is supported by 37 health-related organizations.

The campaign was created in response to the Canadian Adverse Events Study, led by Dr. Ross Baker and Dr. Peter Norton (CMA7 2004;170:1678-86). It found that 1 in 13 Canadian patients was harmed during a hospital stay and an estimated 9250 to 23750 Canadians die - annually after a preventable adverse event in an acute-care hospital. Within the study sample, $7.5 \%$ of patients admitted to hospital in 2000 experienced an adverse event; about $37 \%$ of these were judged to be "highly preventable." Internationally, the incidence of adverse events ranges from $1.9 \%$ to $16.6 \%$ of annual hospital admissions.

The campaign provides interactive tools to help patientcare teams across Canada implement 1 or more of 6 targeted strategies to reduce adverse events. "It's bold. It's an important first step," says Baker, a health policy professor at the University of Toronto.

The 6 strategies are:

- Deploy a rapid response team at the first sign a patient is getting worse.

- Prevent avoidable deaths from heart attacks by delivering reliable, evidence-based care.

- Prevent adverse drug events by double-checking medications with the patient, on their charts and in databases.

- Prevent central line-associated bloodstream infection through a series of evidencebased interventions.

- Prevent surgical site infection by implemented the "SSI bundle" in all surgical patients.
- Prevent ventilator-associated pneumonia by following a series of steps known as the "Ventilator Bundle."

These evidence-based strategies can lead to reduced mortality and adverse events, says Phil Hassen, Institute CEO and campaign chair. The institute also provides tools to track outcomes and measure results through to December 2006.

Baker says the critical issue is how hospitals, which are already stressed in terms of capacity and resources - can implement these changes.

"The success of the plan hinges on providing the backup expertise to the front-line teams," he adds. "To provide assistance in answering questions and help in carrying out the changes in terms of documenting the work and reporting results."

Hassen and Baker say it is too early to estimate the extent to which the plan will reduce adverse events. - Fanis Hass, Ottawa 